\title{
Computer vision techniques for forest fire perception
}

\author{
J.R. Martinez-de Dios ${ }^{\mathrm{a}, 1}$, B.C. Arrue ${ }^{\mathrm{a}, 1}$, A. Ollero ${ }^{\mathrm{a}, 1}$, L. Merino ${ }^{\text {b,* }}$, \\ F. Gómez-Rodríguez ${ }^{\mathrm{a}, 1,2}$
}

a Dpto Ing. de Sistemas y Automática, Escuela Superior de Ingenieros, Universidad de Sevilla, Camino de los Descubrimientos, s/n, 41092 Sevilla, Spain

${ }^{\mathrm{b}}$ Escuela Politécnica Superior, Universidad Pablo de Olavide, Crta. Utrera, km. 1, 41013 Sevilla, Spain

\begin{abstract}
This paper presents computer vision techniques for forest fire perception involving measurement of forest fire properties (fire front, flame height, flame inclination angle, fire base width) required for the implementation of advanced forest fire-fighting strategies. The system computes a 3D perception model of the fire and could also be used for visualizing the fire evolution in remote computer systems. The presented system integrates the processing of images from visual and infrared cameras. It applies sensor fusion techniques involving also telemetry sensors, and GPS. The paper also includes some results of forest fire experiments.
\end{abstract}

Keywords: Perception systems; Infrared and visual image processing; Forest fires; Sensor fusion

\section{Introduction}

Hundreds of millions of hectares are devastated by wildfires each year. Forest fires lead to the destruction of forests and the wildlife that inhabits them and have disastrous social, economic and environmental impacts. Forest fire fighting involves extensive human resources. Fire fighting is a very dangerous activity, which originates causalities every year. In many cases, the lack of information about the current state and the dynamic evolution of fire plays a central role in the accidents.

Thus, forest fire perception in real-time is a key issue for the development of advanced fighting strategies. Today GPS systems make possible to know the current position of the resources. Furthermore, telematic systems integrat-

\footnotetext{
* Corresponding author. Tel.: +34 954348350; fax: +34 954348377.

E-mail addresses: jdedios@cartuja.us.es (J.R. Martinez-de Dios), barrue@cartuja.us.es (B.C. Arrue), aollero@cartuja.us.es (A. Ollero), lmercab@upo.es (L. Merino), gomezro@cartuja.us.es (F. GómezRodríguez).

${ }^{1}$ Tel.: +34 954487349; fax: +34 954487340 .

2 Present address: Dpto Arquitectura y Tecnología de Computadores, Universidad de Sevilla Avda, Reina Mercedes, s/n, 41012 Sevilla, Spain.
}

ing headquarters computers, portable field computers, and communication are able to provide real-time information and to transmit forest fire fighting commands [21]. However, the information about the fire front, flame height, flame inclination angle, fire base width, etc. is very difficult to obtain and involves advanced computer vision systems. It should be noted that forest fire perception suffers from all the general drawbacks that are present in field perception (uncontrollable and sudden changes in environmental conditions, calibration problems, etc.), plus some others related to the particular characteristics of fires: very hostile environment, smoke, and the nature of the fire (non-rigid and motion difficult to be predicted). Artificial vision and image processing techniques have been developed mainly for forest fire detection [15,2,7]. Also, several satellite-based systems have been proposed for forest fire monitoring. However, the time scale and spatial resolution of these systems is still very low for the requirements of forest fire fighting in many cases [19].

This paper presents a new system for forest fire perception involving sensor fusion (i.e. visual, and infrared (IR) cameras, GPS) and several computer vision techniques for real-time measurement and visualization of significant 
properties of forest fires. The presented system is able to compute the location of the fire front in geolocated coordinates, and also characteristics of the fire such as fire base width, flames length, flames inclination angle, maximum flame height, and also the temporal evolution of the fire front in a map. The system has been tested online in many controlled field fire experiments carried out since 1998 and could be used to provide very valuable information in forest fire fighting.

Furthermore, the proposed system can be extended to be used with cameras installed in aerial vehicles, including unmanned aerial vehicles [16,12], in future systems for fire fighting. At this point, it should be noted that the idea of using robots for fire fighting is not new (see for example $[1,3])$. In fact, they can be considered as intervention robots in a very hostile environment. However, the practical application of these robots in forest fire fighting still requires significant research and development efforts.

The paper is organized as follows: Section 2 presents the architecture of the "Experimental Fire Monitoring Tool". Section 3 describes the perception system including the advantages and drawbacks of using different types of cameras and views. Section 4 is devoted to the image processing techniques that has been developed and integrated in the perception system. Section 5 presents some experimental results. Section 6 presents the conclusions and at last acknowledgements.

\section{Experimental Fire Monitoring Tool}

The "Experimental Fire Monitoring Tool" processes synchronized sequences of infrared and visual images to estimate, in real-time, fire propagation features such as rate of spread, flame length, flame height, flame inclination angle, fire base width, and the position of the fire front; and produces and displays views of the 3D fire model built with these measures. Fig. 1 shows a schematic description of some parameters required for forest fire characterization.

The architecture of the proposed system is shown in Fig. 2. The tool has two types of inputs. The first one is

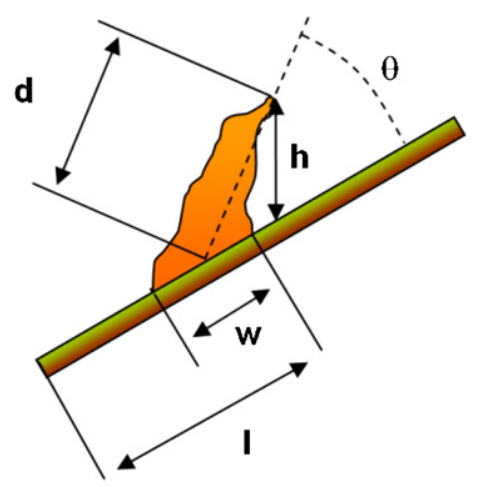

Fig. 1. Description of the fire parameters to be measured: flame length $(d)$, flame height $(h)$, fire front width $(w)$, position of the most advanced point with respect to a reference $(l)$, flame inclination angle $(\theta)$.

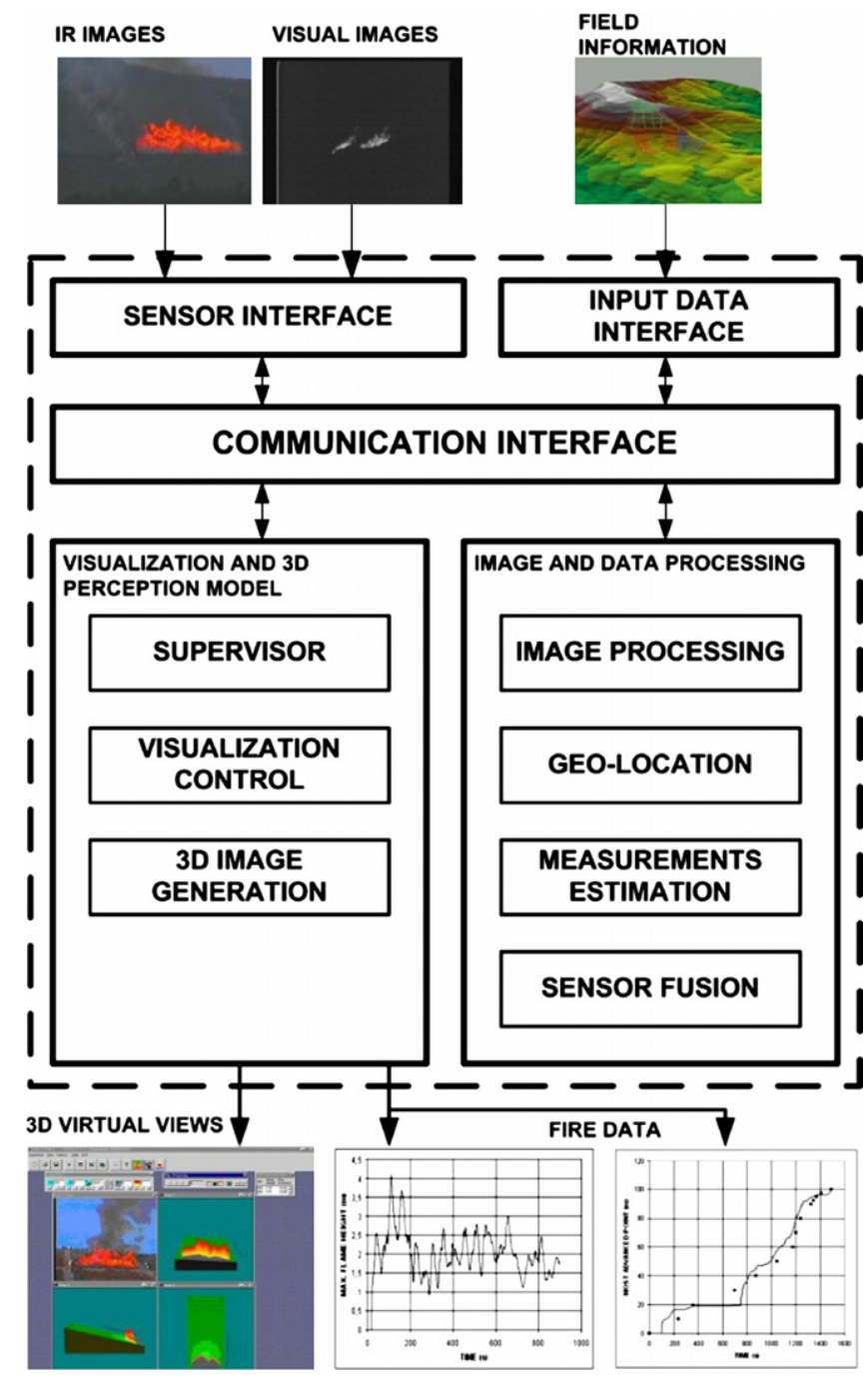

Fig. 2. Architecture of the "Experimental Fire Monitoring Tool".

a group of sequences of infrared and visual images from several views. The real-time image acquisition tool (within the Sensors Interface block) allows several synchronized input sequences taken by cameras at different locations. The other type of inputs is field information, including terrain maps. Also, information regarding the number of cameras deployed and their location (by means of DGPS) and relative orientation with respect to the fire is considered. The system allows versatile combination of different views and cameras.

The main objectives of the Image Processing block, which will be described in detail in Section 4, are to extract the previously mentioned fire features from the images and to obtain measures in real-world coordinates. This block is divided into four sub-blocks: Processing, Geolocation, Measurements Estimation, and Sensor Fusion. The Processing sub-block is responsible for the analysis of each image and obtains estimations on the image plane of fire properties. The Geolocation sub-block combines the results obtained from the Processing sub-block with field data (terrain model and landmarks) to compute real-world estima- 
tions of the fire features. If several cameras are used the Sensor Fusion sub-block implements the combined processing techniques that will be described in Section 4.4. The Measurements Estimation block computes additional information such as the most advanced point of the fire front with respect to a reference, and also implements filtering techniques to eliminate spurious effects and to reduce inaccuracies.

The Visualization and 3D Perception Model block generates and displays the 3D views of the terrain model and the 3D views of the fire model computed from the Measurements Estimation sub-block. The block also displays and stores in files the data computed from the fire.

The Communication Interface is responsible for the information interchange and synchronization between all the blocks.

\section{Deployment of the perception system}

The objectives of the "Experimental Fire Monitoring Tool" were presented in the previous section. The system uses redundant information, given by several cameras at different locations, and considering different modalities (IR and visual cameras), to cope with these objectives.

Fig. 3 illustrates the deployment of several cameras (visual and infrared) from several views. The perception

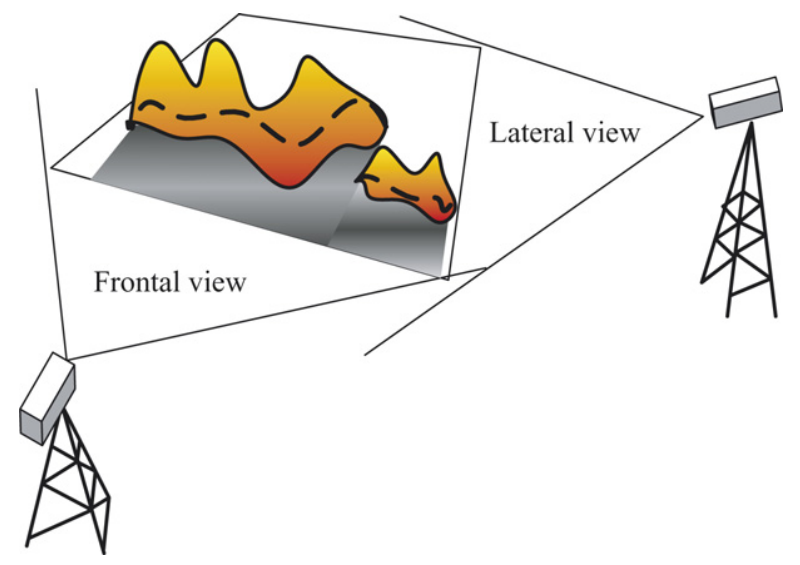

Fig. 3. Scheme of cameras deployment. system described in this paper considers cameras in fixed positions. Fig. 4 shows two views obtained from cameras on the ground.

Fixed cameras can be deployed in a rich variety of combinations such as frontal or backward view (the camera axis is perpendicular to the fire front) and lateral view (the camera axis is parallel to the fire front) as shown, respectively, in Fig. 4a and b. The different positions have advantages and drawbacks. When smoke does not occlude the flames, frontal visual images allow the extraction of the fire front and are able to estimate the rate of spread, but not the flame angle. They can also estimate the flame height. Lateral visual cameras are useful to extract the maximum flame height, flame inclination angle, and fire base width.

By using aerial images, it would be easier to obtain measures of the fire front, including rate of spread and total burnt area as well as the more intense parts of the fire front, which is a valuable information for fire fighting. However, it would be necessary to reduce the effect of the vibrations of the aerial vehicles. This could be done by means of gimbals that provide gyro-stabilization of the sensor platform or by means of further processing [12].

Infrared images can be used to provide estimations of the fire front such as its position, rate of spread, and fire base width. On the other hand, the processing of the images from visual cameras can provide flame measures such as the flame length, flame height over the terrain and flame angle. Although visual cameras have lower costs, sometimes they cannot provide accurate measurements, particularly when smoke occludes the fire. In these cases, IR cameras are very valuable because smoke is transparent for infrared radiation in the spectral band considered (3$5 \mu \mathrm{m})$ [6], and then does not occlude the fire front in the infrared images. However, the adaptation of the IR cameras to the filming of flames (up to $1200^{\circ} \mathrm{C}$ ) sometimes requires attenuators to avoid the saturation of the sensor.

The "Experimental Fire Monitoring Tool" also requires as inputs the position of the cameras and of some landmarks in geographical coordinates (as UTM) in order to geolocate the fire segmented on the image plane (Section 4.3). These positions are measured with DGPS devices.
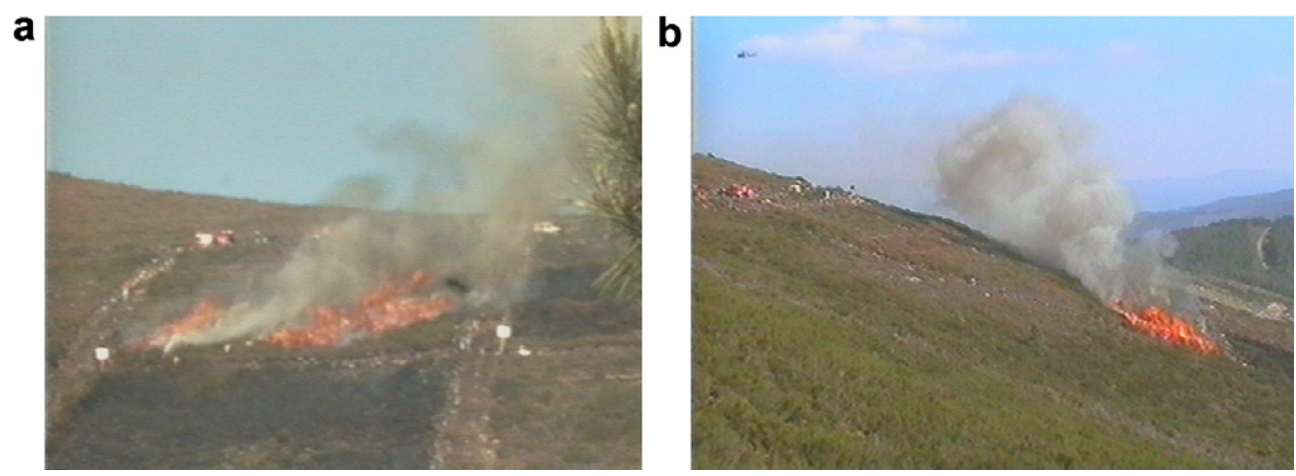

Fig. 4. Frontal (a) and lateral view (b) of one field fire experiment carried out in Gestosa (Portugal) in 1999. 

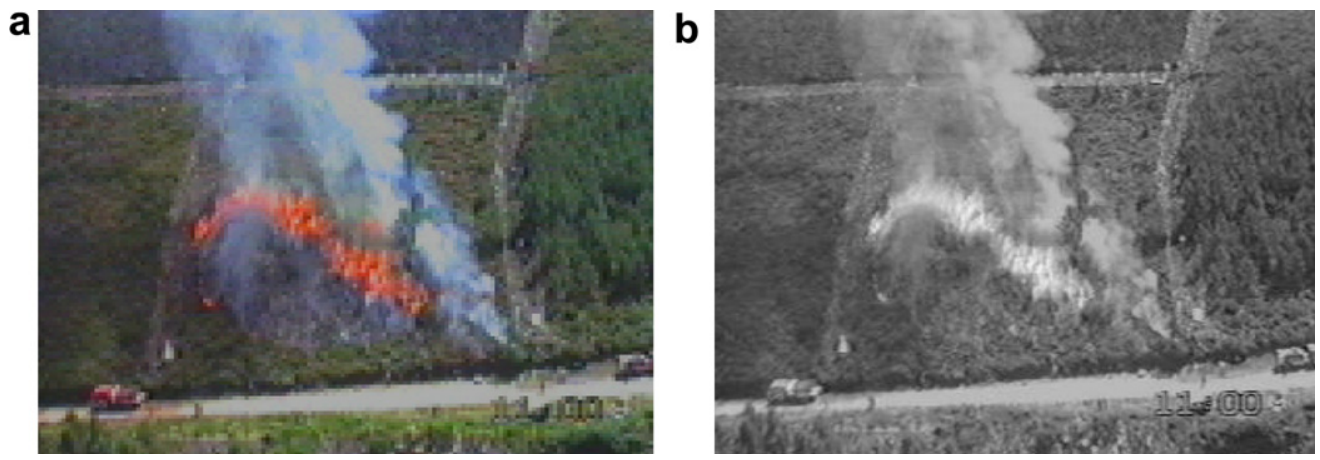

Fig. 5. Colour visual image from a forest fire (a) and its corresponding red component (b)

The presented system can consider a wide variety of camera types and positions. The configuration can be chosen according to the measures needed. Computer vision systems based on multiple cameras obtain complementary and sometimes redundant information, which is useful to reduce errors and increase the reliability of the system, see Section 4.4.

\section{Image processing}

This section is devoted to the description of the algorithms used for extracting the fire measures from the images. The section is divided into five sub-parts: fire segmentation, fire characterization, geolocation, combined processing and filtering.

\subsection{Fire segmentation}

Different segmentation methods are applied depending on the type of image: visual or infrared.

\subsubsection{Visual images}

Two main segmentation techniques have been considered in the "Experimental Fire Monitoring Tool". The first one is based on the fact that visual colour images of fire have high magnitude components in the red component of the RGB coordinates. In fact, in the fire experiments carried out, fire originates the highest magnitude in the red RGB component (see Fig. 5a, which shows a colour visual image from a forest fire, and Fig. 5b, which shows its red component). This property allows using simple thresholdbased criteria. The experiments show that the iterative thresholding algorithm described in [18], applied over the red field, provides good flame segmentation in many cases. However, the robustness of this approach was not satisfactory in case of presence of smoke in the images.

Therefore, a training-based algorithm similar to that described in [17] has been tested. During the training step, a look-up table for the RGB colour space is built. For each triple $(R, G, B)$, this look-up table returns a Boolean value indicating whether this colour represents fire or not. The training phase builds this look-up table from pairs composed by a colour image and a Boolean mask of the same size of the image. Each position of the mask indicates if the corresponding pixel is fire or background. An RGB histogram is created by accumulating Gaussian distributions centred at the RGB triples corresponding to fire pixels in the image, and subtracting another (smaller) Gaussian distribution centred at the RGB triples corresponding to background. Finally, this RGB histogram is thresholded, resulting in the RGB look-up table.

Both techniques generate binary images with fire regions. Binary erosion is performed to eliminate isolated spurious pixels detected as fire. The final step applies of a region-growing algorithm using colour properties.

\subsubsection{Infrared images}

Infrared cameras generate $\mathrm{B} \& \mathrm{~W}$ images with estimations of the radiation intensity in the scene. In fire images, fire is the source that originates the highest radiation intensity. Thus, it is also reasonable to consider thresholding for segmenting IR images.

For IR images in the band $(3-5 \mu \mathrm{m})$ the radiation intensity of the fire base is higher than that of the flames. Thus, the intensity values of the pixels corresponding to the flames are lower than the intensity values of the pixels corresponding to the fire base, and then a threshold value could be determined to differentiate flames and fire base. The computation of this threshold value is highly application-dependent and the classical threshold selection algorithms tested have shown poor performance in this application.

For IR images a fuzzy multiresolution algorithm is used. This algorithm computes the threshold using heuristic knowledge incorporated in a fuzzy decision system that supervises a multiresolution wavelet-based coarse-to-fine threshold search analysis. An initial histogram region for the intensities of the object is selected by using a coarse description of the image histogram. This initial histogram region is restricted more and more at increasing level of scale under the supervision of fuzzy supervising decision systems that incorporate heuristic knowledge on the type of object and illumination conditions. Fig. 6 shows a scheme of the fuzzy multiresolution method. At each level of resolution the Fuzzy Region Selection System selects the histogram modes likely to be originated by the object of 


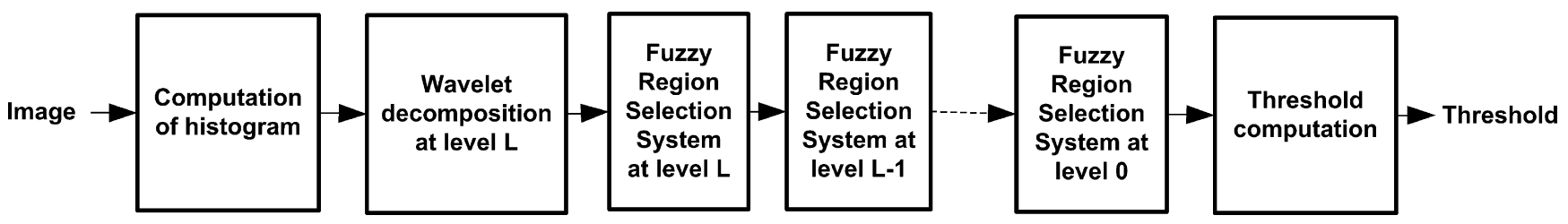

Fig. 6. General scheme of the method fuzzy-wavelet algorithm for threshold selection.

a

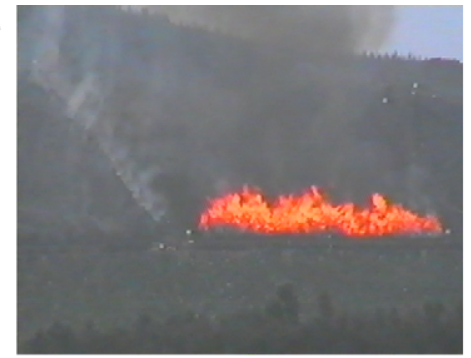

d

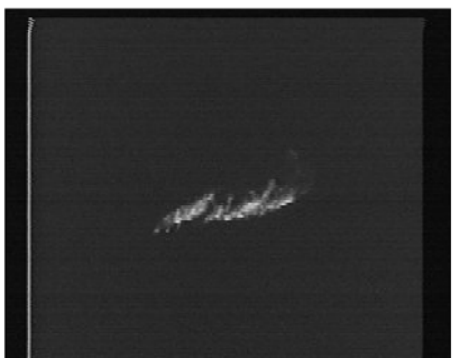

b

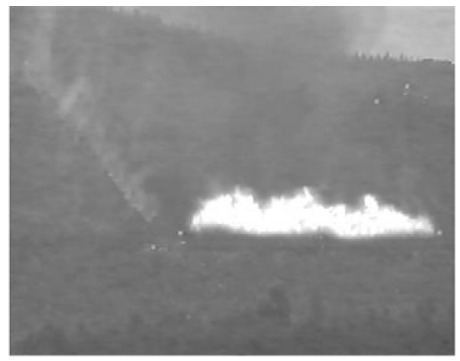

e

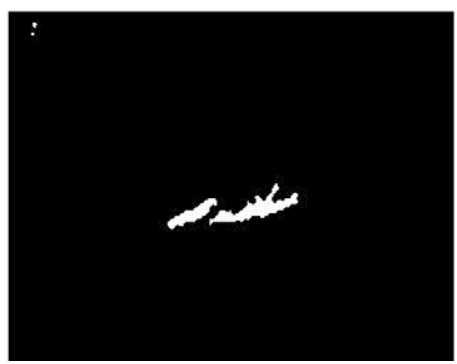

C
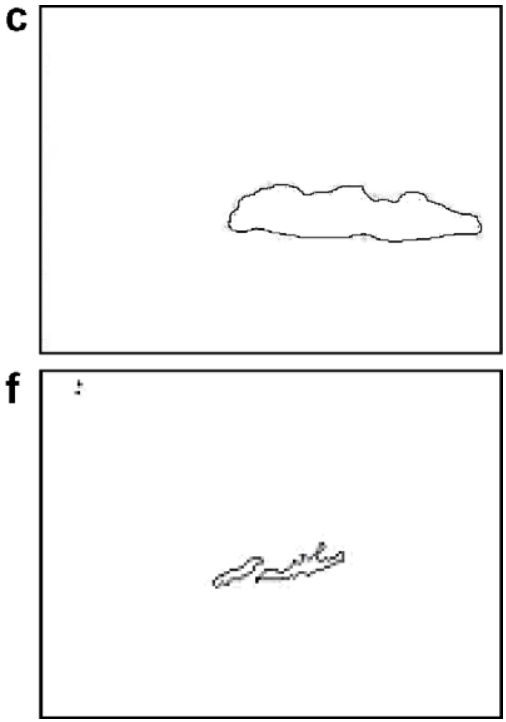

Fig. 7. Application of the algorithms to two fire images: (a) frontal visual image, (b) its red field, (c) contour obtained; (d) an infrared image from a static camera on the ground, (e) thresholded infrared image, (f) contour obtained.

interest. These histogram modes will be analysed with more details at the next (finer) level of resolution. The heuristic knowledge about the objects of interest and the computer vision problem is incorporated into the Fuzzy Region Selection System as a set of fuzzy rules. A more detailed description can be found in $[10,11]$.

Due to the significant level of noise in the images, image thresholding can originate spurious effects, mainly originated by isolated flame pixels in the background and gaps in the flames. A binary correction algorithm was applied to filter out these spurious effects and increase the uniformity of the image. For both type of images, the final step applies contour detection functions to identify the edges of the segmented regions. Once the contours have been computed it is simple to make measurements of the position of fire front, flame height and fire base width.

Fig. 7 illustrates the performance of the above-mentioned algorithms on two images from controlled fire experiments. Two cases are shown: visual image and infrared image. For each case, three images are displayed. The first column (Fig. $7 \mathrm{a}$ and d) shows the original images. Fig. $7 \mathrm{~b}$ shows the red field for the visual image. Fig. 7e shows the thresholded infrared image. Fig. $7 \mathrm{c}$ and $\mathrm{f}$ shows the resulting images after segmentation and contour detection.

\subsection{Fire object characterization}

The results from the previous techniques are fire regions segmented on the image plane. To obtain fire measures the
"Experimental Fire Monitoring Tool" characterizes the segmented fire regions as depicted in Fig. 8. The important characteristics are the flame contour and the fire base (delimited by the forward and backward base contours).

Given a fire region $o$, let $C_{o}^{\mathrm{b}}=\left\{\mathbf{p}_{o k}^{\mathrm{b}}, k=1, \ldots, N_{o}\right\}$ be the set of pixels of the backward base contour of fire region $o . C_{o}^{\mathrm{f}}=\left\{\mathbf{p}_{o k}^{\mathrm{f}}, k=1, \ldots, N_{o}\right\}$ is the set of pixels of the forward base contour and $C_{o}^{1}=\left\{\mathbf{p}_{o k}^{1}, k=1, \ldots, N_{i}\right\}$ is the set of pixels of the flame contour. In general, depending on the relative orientation of the camera with respect to the fire-only part of the backward or forward base contours will be seen (the flames will occlude part of them).

The dynamic characteristics of fire are used to characterize the fire regions. In general, the positions of the fire base

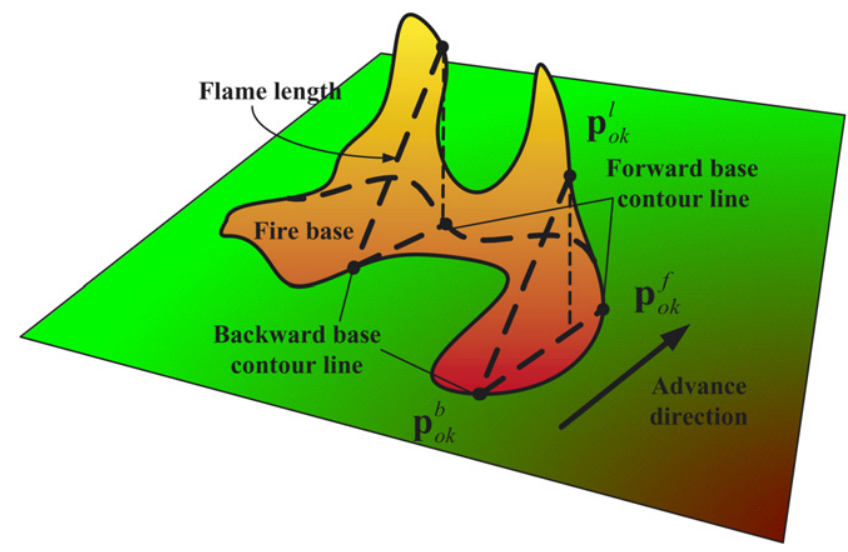

Fig. 8. Scheme of characterization of fire regions. 
pixels change more slowly than those of the flame pixels. The binary image $\hat{I}_{i}$ contains the pixels that have not changed over the last $n$ frames:

$\hat{I}_{i}=I_{i-n} \otimes I_{i-n+1} \otimes \cdots \otimes I_{i}$,

where $\otimes$ represents the binary AND operator and $I_{i}$ the binary image containing the fire objects at time $i$. If $n$ is correctly chosen, $\hat{I}_{i}$ contains only fire base pixels. The edge of the fire regions in $\hat{I}_{i}$ represents the contours of the fire base $\left(C_{o}^{\mathrm{f}} \cup C_{o}^{\mathrm{b}}\right)$.

A further characterization is needed to compute the fire base width, identifying the pixels of the fire base corresponding to the forward and backward base contours. That can be done by analyzing the motion of the fire base. The velocity $\overline{\mathbf{v}}_{o, i}^{\mathrm{b}}$ of the centroid of $C_{o}^{\mathrm{f}} \cup C_{o}^{\mathrm{b}}$ (fire base contour for region $o$ ) gives an estimation of the direction of advance of the fire base on the image plane at time $i$. Using the direction of $\overline{\mathbf{v}}_{o, i}^{\mathrm{b}}$, it is possible to differentiate between pixels of the backward base contour and of the forward base contour, and thus it is possible to compute the width in pixels of the fire base (see Fig. 9). To obtain a robust estimation of the temporal evolution of $\overline{\mathbf{v}}_{o, i}^{\mathrm{b}}$, it is averaged along several frames.

Also, it is needed to estimate the flame inclination angle on the image plane in order to assign the pixels of the fire base contour to their corresponding pixels of the top of the flames. A rough estimation is given by vector $\overline{\mathbf{v}}_{o, i}^{1}=\overline{\mathbf{c}}_{o, i}^{1}-\overline{\mathbf{c}}_{o, i}^{\mathrm{b}}$, where $\overline{\mathbf{c}}_{o, i}^{1}$ is the centroid of $C_{o}^{\mathrm{l}}$ (the set of pixels corresponding to the top of the flames), and $\overline{\mathbf{c}}_{o, i}^{\mathrm{b}}$ is the centroid of $C_{o}^{\mathrm{b}}$ at time $i$ (see Fig. 9). It should be noted that the inclination angle is not really uniform along the whole fire front, but this approximation is valid for most of the experiments.

Fig. 10 shows an example with three consecutive visual images. The middle row shows the fire base identified with $n=3$ using a flame rate of 1 frame/s. The bottom row

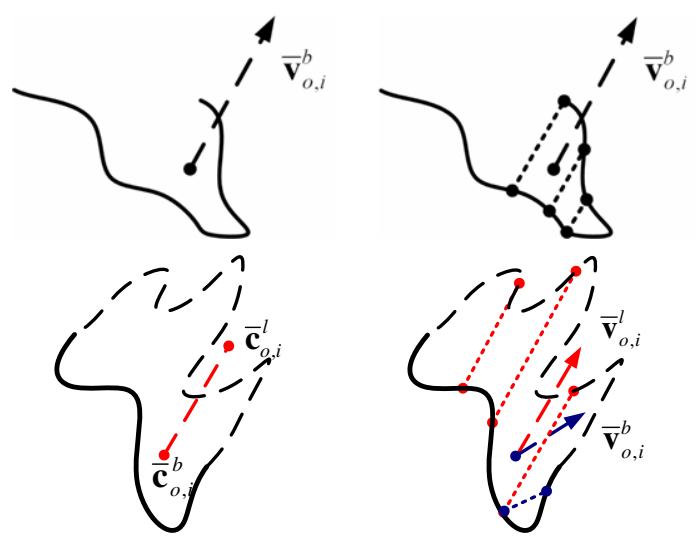

Fig. 9. Top left: fire base contour and direction of advance of its centroid Top right: correspondence among pixels of the backward and forward base contours to compute the fire base width. Bottom left: base contour (solid), top of the flames (dashed) and their centroids. Bottom right: correspondence among pixels of the backward base contour and pixels of the top of the flames. shows in blue the pixels of the backward base contour and in red the pixels of the flame contour.

\subsection{Geo-location}

As it has been pointed out, the image processing block provides image features of the fire, such as the position in pixel coordinates of the fire front, the flame height, and others. The fire front is then geo-located in order to obtain measures of its evolution. This implies that the position and orientation of the camera should be known, and also that the camera should be internally calibrated. All these data should be computed for the set of static cameras deployed in the field (in general, by matching landmarks with their projections on the image plane). In operational conditions, it is interesting to simplify the operations needed to obtain these parameters.

Two basic situations are considered: 2D terrains (i.e., the terrain shape can be approximated by a plane), and full 3D terrains.

\subsection{1. $2 D$ terrains}

If the terrain is planar, the relation between the terrain plane and the image plane is an invertible linear projective mapping, a homography [8]. Without loss of generality, if the plain corresponds to $Z=0$, then the following relations hold:

$$
\begin{aligned}
& s\left[\begin{array}{l}
u \\
v \\
1
\end{array}\right]=\mathbf{A}_{j}\left[\begin{array}{lll}
\mathbf{r}_{1, j} & \mathbf{r}_{2, j} & \mathbf{t}_{j}
\end{array}\right]\left[\begin{array}{c}
X \\
Y \\
1
\end{array}\right]=\mathbf{H}_{j}\left[\begin{array}{c}
X \\
Y \\
1
\end{array}\right], \\
& \mathbf{A}_{j}=\left[\begin{array}{lll}
\alpha_{j} & \gamma_{j} & u_{0, j} \\
0 & \beta_{j} & v_{0, j} \\
0 & 0 & 1
\end{array}\right],
\end{aligned}
$$

for camera $j$, where $\mathbf{p}=\left[\begin{array}{lll}u & v & 1\end{array}\right]^{\mathrm{T}}$ are the homogeneous pixel coordinates of the point $\mathbf{P}=\left[\begin{array}{llll}X & Y & 0 & 1\end{array}\right]^{\mathrm{T}}$ in world coordinates. $\mathbf{r}_{1, j}, \mathbf{r}_{2, j}$, and $\mathbf{t}_{j}$ are, respectively, the two first columns of the rotation matrix and the translation vector which relate the world coordinate system to a coordinate system centred at camera $j$ (see Fig. 11), and $\mathbf{A}_{j}$ represents the internal calibration for camera $j$. This situation is interesting because only four correspondences among points and their image coordinates are needed to compute the homography $\mathbf{H}_{j}$. Also, there is no need to compute explicitly the external or internal parameters of the camera. The homography is invertible. Therefore, if $\mathbf{H}_{j}$ is known, it is possible to calculate the geolocated coordinates of every pixel on the image plane.

It should be noted that, although the terrain may be not planar, if the cameras are located far enough (i.e., if the distance from the camera to the terrain is large compared to the deviations from a planar surface), the planar approximation is still valid, and that is the case in many of the experiments carried out. Notice that in the case of forest fires it is not likely to deploy the cameras very close to the fire. 

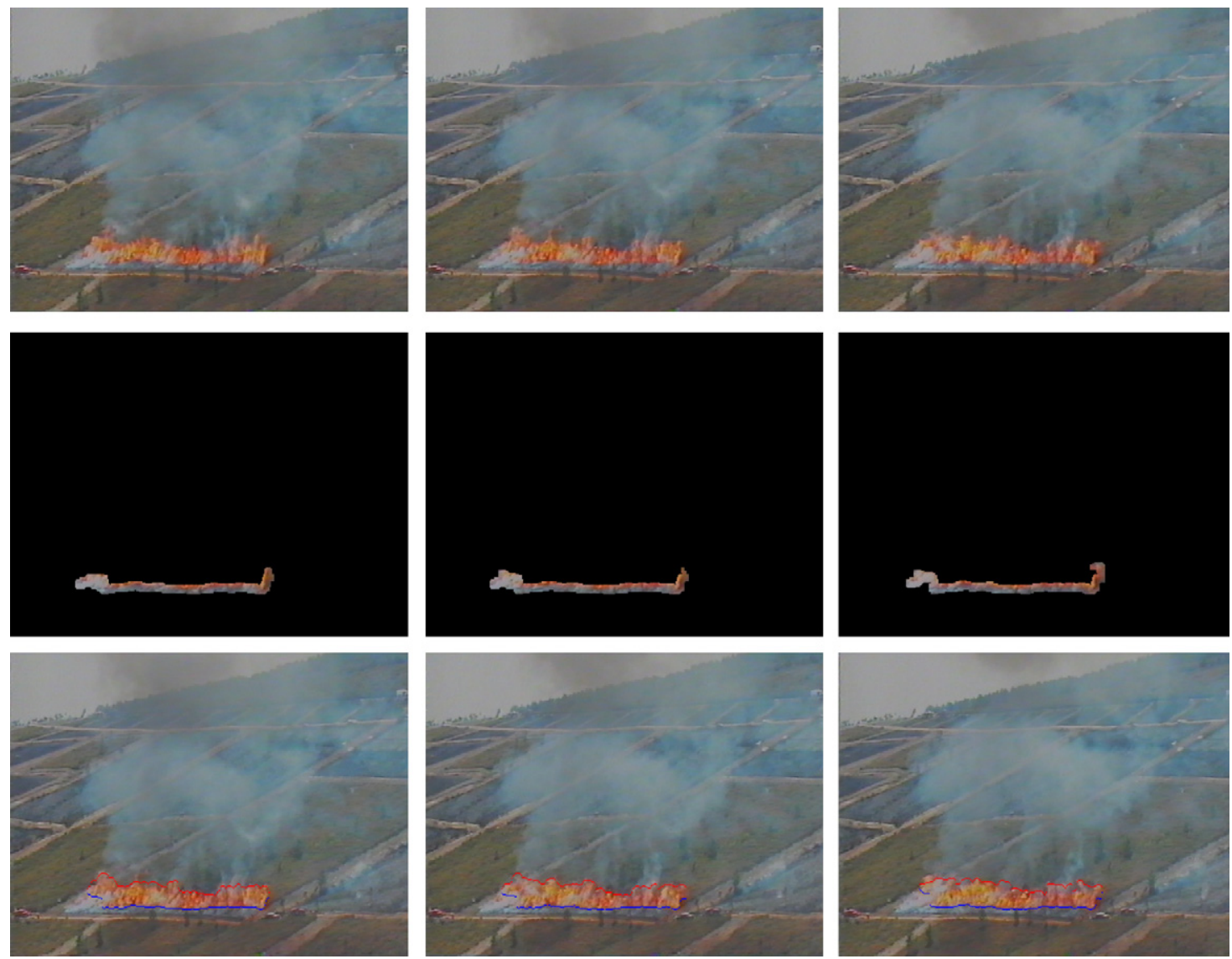

Fig. 10. Top row: three consecutive images. Middle row: fire base pixels identified with $n=3$ and fame rate: 1 frame/s. Bottom row: in blue, pixels of the backward base contour and in red, pixels of the flame contour.

\subsection{2. $3 D$ terrains}

In this case, a digital elevation model of the terrain is used to represent the scene. Geolocation of fire front pixels, which are located on the ground, is done by projecting the pixels on the terrain model, see Fig. 11. In this case, the camera should be explicitly calibrated. Relations (4) and (5) become:

$s\left[\begin{array}{c}u \\ v \\ 1\end{array}\right]=\mathbf{A}_{j}\left[\begin{array}{llll}\mathbf{r}_{1, j} & \mathbf{r}_{2, j} & \mathbf{r}_{3, j} & \mathbf{t}_{j}\end{array}\right]\left[\begin{array}{c}X \\ Y \\ Z \\ 1\end{array}\right]=\mathbf{P}_{j}\left[\begin{array}{c}X \\ Y \\ Z \\ 1\end{array}\right]$

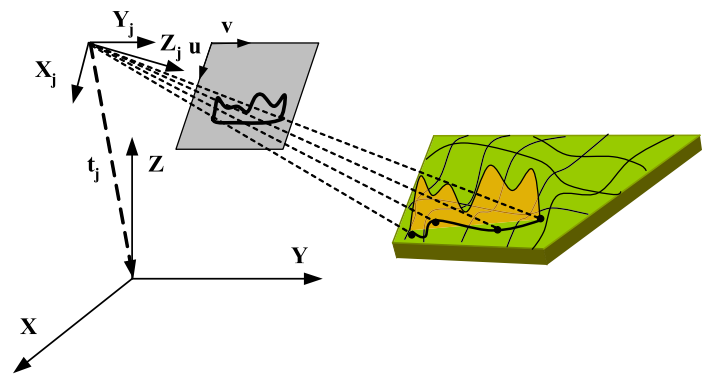

Fig. 11. Scheme of image projection on the terrain.
Several correspondences between pixels on the image plane and 3D points are needed to calibrate the camera. In general, it is not realistic to know the position of many points or landmarks in forest fires scenes. Thus, the following assumptions are made to reduce the number of correspondences required for calibration:

- The position of the camera is measured by means of DGPS.

- The rotation matrix is parameterized using the row, pitch and yaw angles, and the roll angle is considered as 0 .

- The skew $\gamma_{j}$ is considered 0 , and the aspect ratio 1 $\left(\alpha_{j}=\beta_{j}\right)$. The principal point $\left[u_{0, j} v_{0, j}\right]^{\mathrm{T}}$ in (5) is also assumed to be known.

Artificial beacons (Fig. 12) and natural marks (such as a tree or a fire fighter truck) were used for calibration in the fire tests shown in Section 5. The calibration procedure is described in Section 4.3.3.

\subsubsection{Iterative and non-lineal calibration method}

This technique is used to compute the orientation of the camera and its focal length. The orientation is expressed using the roll, pitch, and yaw (RPY) angles. As mentioned, 


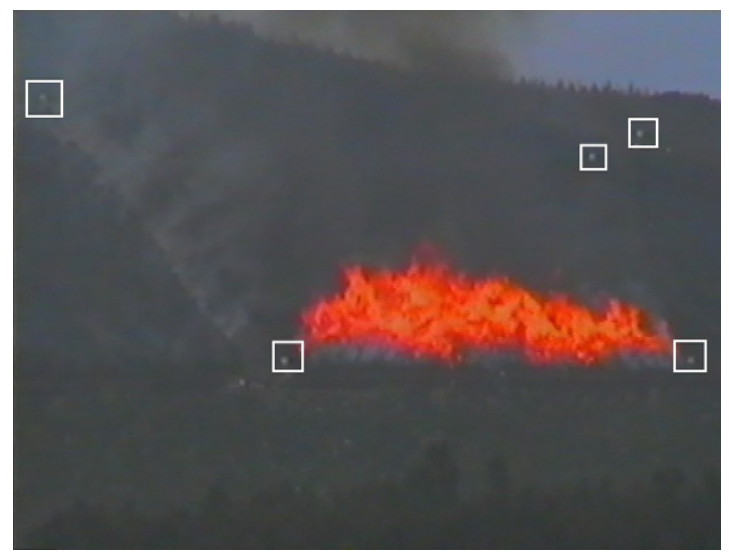

Fig. 12. Landmarks used during the experiments.

this technique assumes that the roll angle is zero (a usual assumption for fixed cameras), and the UTM coordinates of the position of the camera, as well as the UTM coordinates of natural landmarks or beacons placed on the terrain, are known. Then, the parameters to be computed are: the yaw $\left(\varphi_{Y}\right)$ and pitch $\left(\varphi_{X}\right)$ angles, and the focal length $\left(\alpha_{j}=\beta_{j}\right)$.

The method works as follows: taking initial values of the calibration parameters $\left(\varphi_{Y}, \varphi_{X}\right.$, and $\left.\alpha_{j}\right)$, the transform matrix $\hat{\mathbf{P}}_{j}^{0}$ is estimated. Then, errors are obtained by projecting the landmarks on the image plane using $\hat{\mathbf{P}}_{j}^{0}$ and comparing their positions with the real values on the image. The method will change the parameters trying to minimize these errors, obtaining a new estimation $\hat{\mathbf{P}}_{j}^{k}$ in step $k$. The procedure follows until the errors are below a certain level.

The initial values of $\varphi_{Y}$ and $\varphi_{X}$ in the iterative algorithm are the average of the angles that form each landmark with the camera position (in order to have a suitable initial approximation, these landmarks should be well distributed over the image). In the experiments described in this paper, this average is roughly the centre of the area to be burned. This is a coarser estimation but it is sufficient as the first values for the algorithm.

The algorithm optimizes the parameters separately with a non-linear least squares optimization procedure based on the interior-reflective Newton method [4,5] until the errors are lower than a threshold value.

\subsection{Combined processing}

By using the previous relations, it is possible to obtain fire measures in geographical coordinates from the features obtained from the image planes. As it has been pointed out in Section 2, the "Experimental Fire Monitoring Tool" allows several input sequences, which can be used to improve the measures obtained. This can be approached in two ways.

The first approach could be seen as a decision-level sensor fusion [9]. Some fire parameters can be measured from several cameras independently. In this case, each camera $j$ could be seen as a measurement device, that generates a measure $m_{j i}$ at time $i$. There is a confidence index $\omega_{j i}$ associated to this measure. The measures are combined following the equation:

$M_{i}=\frac{\sum_{j} m_{j i} \omega_{j i}}{\sum_{j} \omega_{j i}}$

The confidence indexes are computed as:

$\omega_{j i}=F_{j}\left(\frac{1}{r_{j i}^{2}}\right)$,

where $F_{j}$ encodes the heuristical information related to the camera $j\left(F_{j} \in[0,1]\right)$, and $r_{j i}$ is the resolution (meters/pixel) associated to the measure at time $i$ for camera $j$. As an example, if $m$ is the advance of the fire front with respect to a reference, $F$ will be set to the maximum value for infrared cameras and a lower value for visual cameras, due to the fact that the infrared cameras considered are not affected by smoke (see Section 3). The resolution $r_{j i}$ is computed by using the calibration information related to camera $j$.

Similar expressions to Eqs. (7) and (8) could be obtained from a Bayesian approach by considering the measures as independent and assuming a normal distribution for the errors in $m$, of zero mean and a standard deviation proportional to the resolution $r$, and obtaining the most likely value given the different measures [14].

However, the processing of a single image sequence does not usually allow computing all the parameters of the fire, due to the geometry of the camera with respect to the fire or due to the characteristics of the image. For instance, the fire base width cannot be measured with a single visual camera with frontal view due to the occlusion by the flames. Also, an infrared image does not allow estimating the flame height with accuracy because the flame radiation decreases when the distance to the fire front base increases. Moreover, the inclination of the flames has to be computed using lateral views.

Another approach could be seen as a sensor fusion technique at the feature level. The features estimated in one image can be represented on the image plane of other camera by applying view transformations as those described in Section 4.3. Given two cameras, $i$ and $j$, a point $\mathbf{P}$ is observed as $\mathbf{p}^{i}$ in camera $i$ and as $\mathbf{p}^{j}$ in camera $j$. For 2D terrains, considering homogeneous coordinates, the relation between cameras is (see example in Fig. 13):

$\mathbf{p}^{j}=\mathbf{H}_{j} \mathbf{H}_{i}^{-1} \mathbf{p}^{i}$,

where $\mathbf{H}_{j}$ (and $\mathbf{H}_{i}$ ) is given by Eq. (4). In case of 3D scenes and for calibrated cameras, a pixel $\mathbf{p}^{i}$ in camera $i$ can be projected over the terrain, and then re-projected on the image plane of camera $j$ :

$\mathbf{p}^{j}=\mathbf{P}_{j} \mathbf{P}$,

where $\mathbf{P}_{j}$ is defined by Eq. (6). 

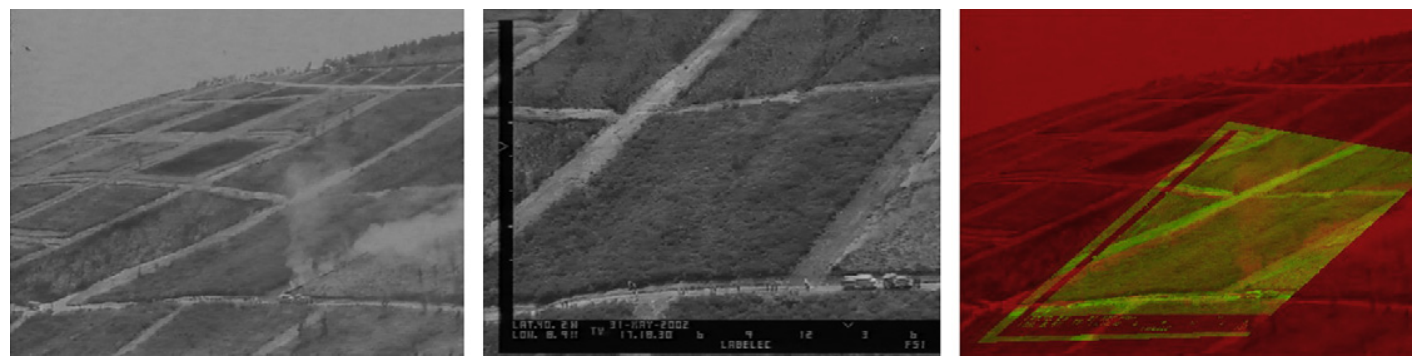

Fig. 13. Left: one view of the terrain. Middle: same terrain viewed from a frontal camera. Right: both views overlapped in the same image using relation (7).

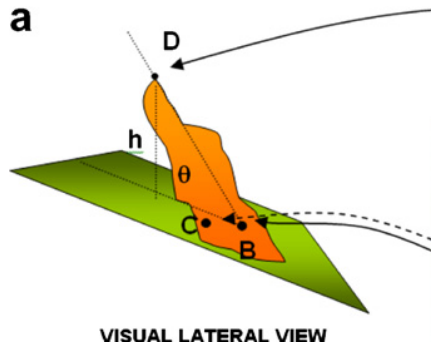

VISUAL LATERAL VIEW
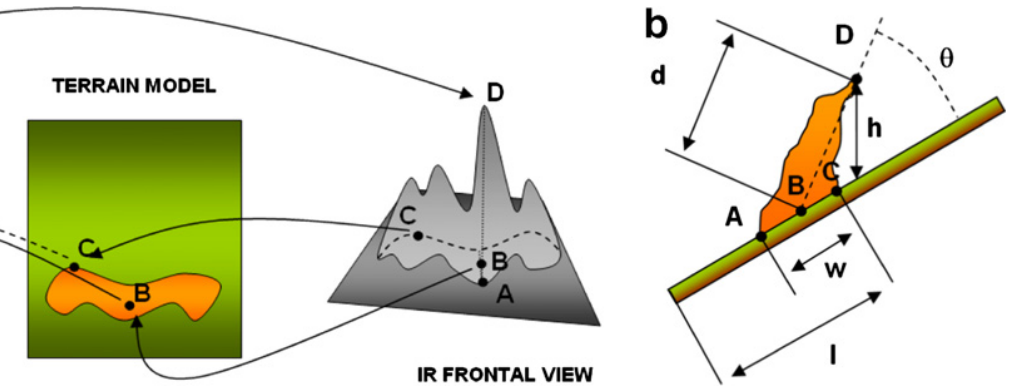

Fig. 14. (a) Example of combined IR-visual processing using different views, (b) scheme with geometrical parameters of fire.

Fig. 14a shows an example of combined image processing using an infrared frontal view and a lateral visual view for a fire spreading on a planar surface. From the lateral visual image, it is not possible to determine with accuracy the most advanced point of the fire front with respect to the ignition line (parameter $l$ in Fig. 14b), because the flames occlude part of the forward base contour (point $\mathbf{C}$ in Fig. 14a, lateral view). However, $\mathbf{C}$ can be clearly obtained from the segmentation of the fire base (Section 4.1.2) in the infrared frontal view (C in Fig. 14a, IR view). The fire base width can be computed by using the infrared image as well. The flame inclination angle ( $\theta$ in Fig. 14b) can be computed in the lateral view using the procedure described in Section 4.2 (the IR view cannot provide a reliable measure in this case). However, a more accurate angle can be obtained if the point in the fire base corresponding to the maximum height flame (point $\mathbf{B}$ ) is obtained using the IR view. Finally, this point $\mathbf{B}$ can be transformed into the lateral view. Then, the parameters $\theta$ and $h$ (see Fig. 14b) can be easily computed.

The case of a frontal visual view and a lateral visual view is similar, but now the frontal view only provides point $\mathbf{A}$ in the fire base corresponding to the highest flame (it cannot provide the most advanced point $\mathbf{C}$ as mentioned before). Transforming point $\mathbf{A}$ into the lateral view, the flame height $h$ and an approximate value of the inclination $\theta$ can be measured.

\subsection{Filtering}

A wide range of local errors and perturbations are frequently originated in visual monitoring and measurement in unstructured environments. These perturbations often generate unnatural local evolution of the target monitored with harmful consequences in many applications. In forest fires, these perturbations have the global effect of originating negative increments in the advance of the fire front, which is not natural due to the absence of unburned material.

A fuzzy-wavelet technique was adopted to filter out these perturbations. The main idea is to design a filter able to eliminate the perturbations from the noisy input at an elementary scale of resolution. Fuzzy filters are proposed for its simplicity of adaptation to specific applications. This filter is applied to the wavelet decompositions of the noisy input sequence at several levels of resolution, in order to eliminate the perturbations at those levels of scale. This fuzzy-wavelet technique is capable of eliminating local perturbations preserving the global trends of the input.

Fig. 15a shows the scheme of the method. Let $s(n)$ be a noisy sequence. The algorithm applies the Fuzzy-Wavelet Filter Component from level $N$ downwards $(N, N-1, \ldots$, $0)$. Fig. 15b shows the scheme of the Fuzzy-Wavelet Filter Component at level $w$. The first step is to apply the wavelet packet decomposition at level $w$ to the input sequence, generating $D_{w, i}$, where $i=\left[0,2^{w}-1\right]$ (see Fig. $15 \mathrm{c}$ ). Then, all decompositions are filtered using the Fuzzy Filter. The resulting sequences, $D f_{w, i}$, are finally combined to reconstruct the sequence.

The Fuzzy-Wavelet Filter Component at level $w$ cancels the undesired perturbations in the neighbourhood $M^{w}$, where $M$ is the number of neighbouring values. First, the scheme shown in Fig. 15a is applied for $N=0$. Therefore, the Fuzzy Filter is applied to $D_{0,0}=s(n)$ and local perturbations within $M$ neighbours are cancelled. If no undesired behaviour is detected in $D f_{0,0}$, the algorithm stops. Other- 

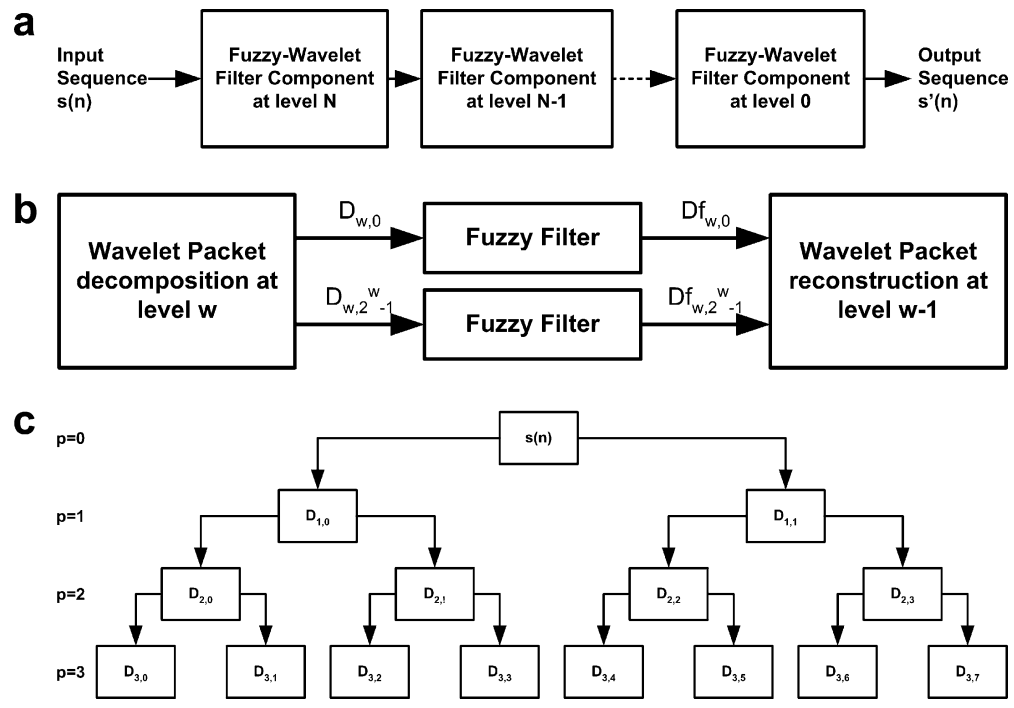

Fig. 15. Fuzzy-wavelet filter: (a) general scheme, (b) Fuzzy-Wavelet Filter Component at level w, (c) wavelet packet decomposition tree.
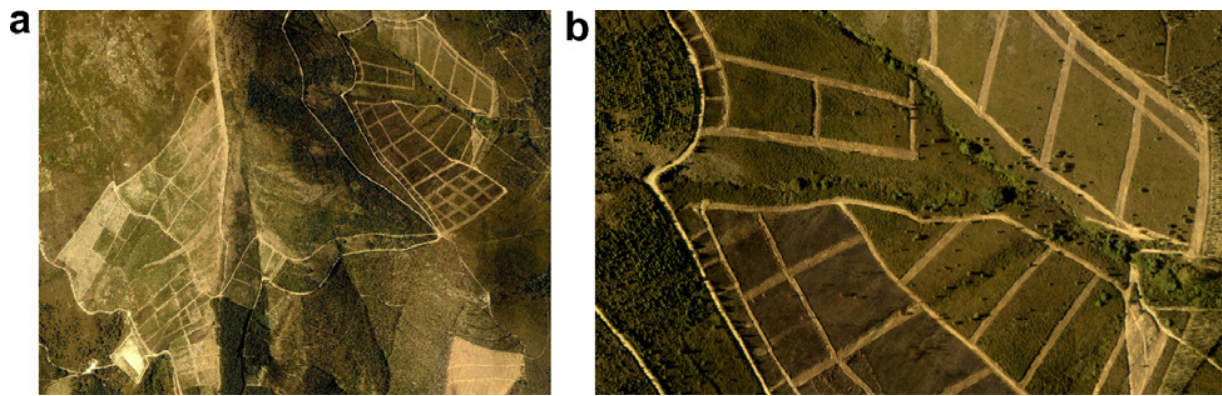

Fig. 16. Left: aerial view of the Gestosa experimental site. The image covers an area of about $4 \mathrm{~km} \times 4 \mathrm{~km}$. Right: zoom of one of the burning areas.

wise, the general scheme should be consecutively applied for $N=1,2, \ldots$ until no harmful effects are found.

The Fuzzy Filter proposed for fire monitoring is based on fuzzy filters based on IF-THEN-ELSE fuzzy reasoning. The operation is window based: a set of two neighbouring values (i.e. $M=3$ ) is considered. Let $x(n)$ be the value in a noisy sequence. The inputs of the fuzzy filter are the increments that take place in the neighbourhood, $\Delta x_{1}=x(n)-x(n-1)$ and $\Delta x_{2}=x(n+1)-x(n)$. The filter processes the inputs using fuzzy rules with the aim of cancelling negative increments. The output, $\Delta y(n)$, is the correction term to cancel negative increments, i.e. the corrected value is $y(n)=x(n)+\Delta y(n)$. If no rule is activated, the central value is left unchanged. The fuzzy filters can be easily particularised to the elimination of negative increments of the fire front spread.

\section{Experimental results}

The fire perception system proposed in this paper has been used to monitor field fire experiments carried out in Gestosa (Portugal, see Fig. 16) from 1998 to 2005 [20]. Fig. 17 shows the monitoring tool using two visual cameras (one frontal view and one lateral view) and one infrared camera. A 3D view of the fire model is generated using

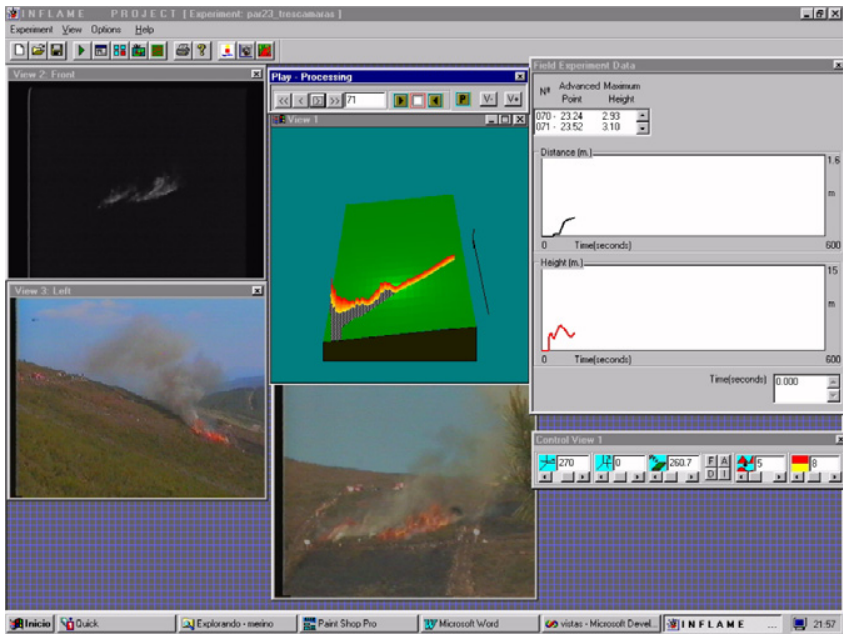

Fig. 17. Monitoring of a fire with two visual cameras and one infrared camera.

the computed measurements and displayed. Fig. 18 shows the monitoring of a fire using only one visual camera. The system provides the fire measures described in precedent sections as well as the rate of spread. The system is also capable of giving the temporal evolution of fire data. 


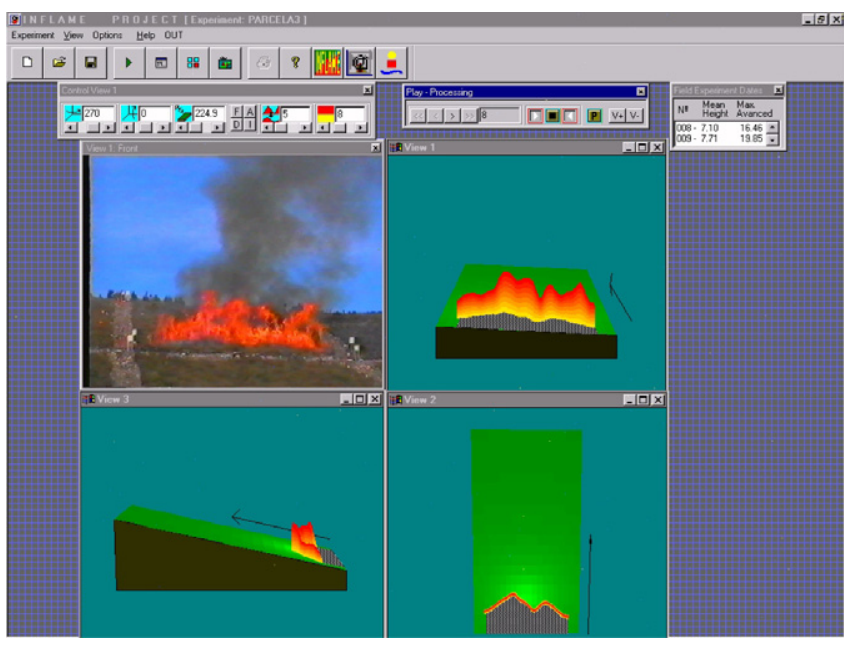

Fig. 18. Monitoring of a fire with one frontal static visual camera.

The fire characteristics provided by the system have been evaluated and compared with results obtained by using classical fire measuring methods. An independent estimation of the fire front evolution was obtained by deploying threads on the field and writing down the time instants when the threads were reached by the fire [20]. The results of the evaluations carried out were satisfactory, obtaining very low errors. Fig. 19 shows the temporal evolution of the most advanced point of the fire front (with respect to the ignition line) of an experiment in May 2000. The upper and lower curves show the results obtained from the analysis of the infrared and visual images respectively. The central one is the result of the sensor fusion described. The dots represent the time instants in which the threads were cut. These results show the synergies obtained by the joint processing and data fusion. Fig. 20 shows the evolution of the fire front in geographical coordinates.

Fig. 21a and $\mathrm{b}$ shows the temporal evolution of the advance of the fire front and the fire front width of an experiment in May 1999. Again, the measurements of the fire front advance are compared to those obtained by

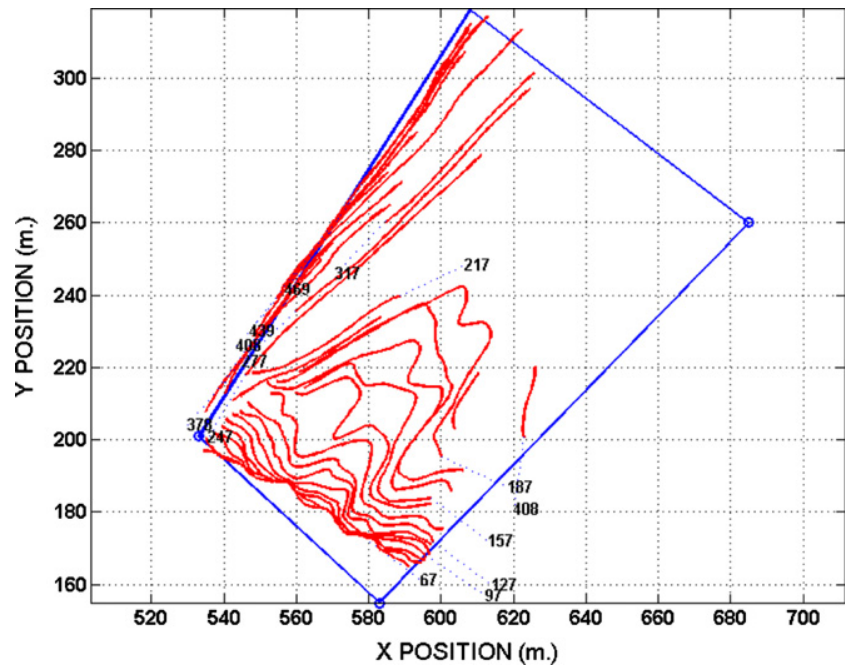

Fig. 20. Nadir view of the fire-front shape evolution in geographical coordinates each $10 \mathrm{~s}$ for the experiment in Fig. 19.

threads deployed on the field. Additional results can be found in [20].

\section{Conclusions}

Forest fires have disastrous impacts. Forest fire fighting involves extensive human resources. It is a very dangerous activity, in which there are many casualties every year. Fire perception in real-time is a key issue for the protection of people and development of fighting strategies.

This paper has presented a system for forest fire perception involving measurement of forest fire properties (fire front location, flame height, flame inclination angle, fire base width) required for the implementation of advanced forest fire-fighting strategies. The design of the computer vision systems involving infrared and visual cameras is discussed. The filters and computer vision techniques, involving algorithms, calibration methods and combined infrared and visual image processing are summarized. The system
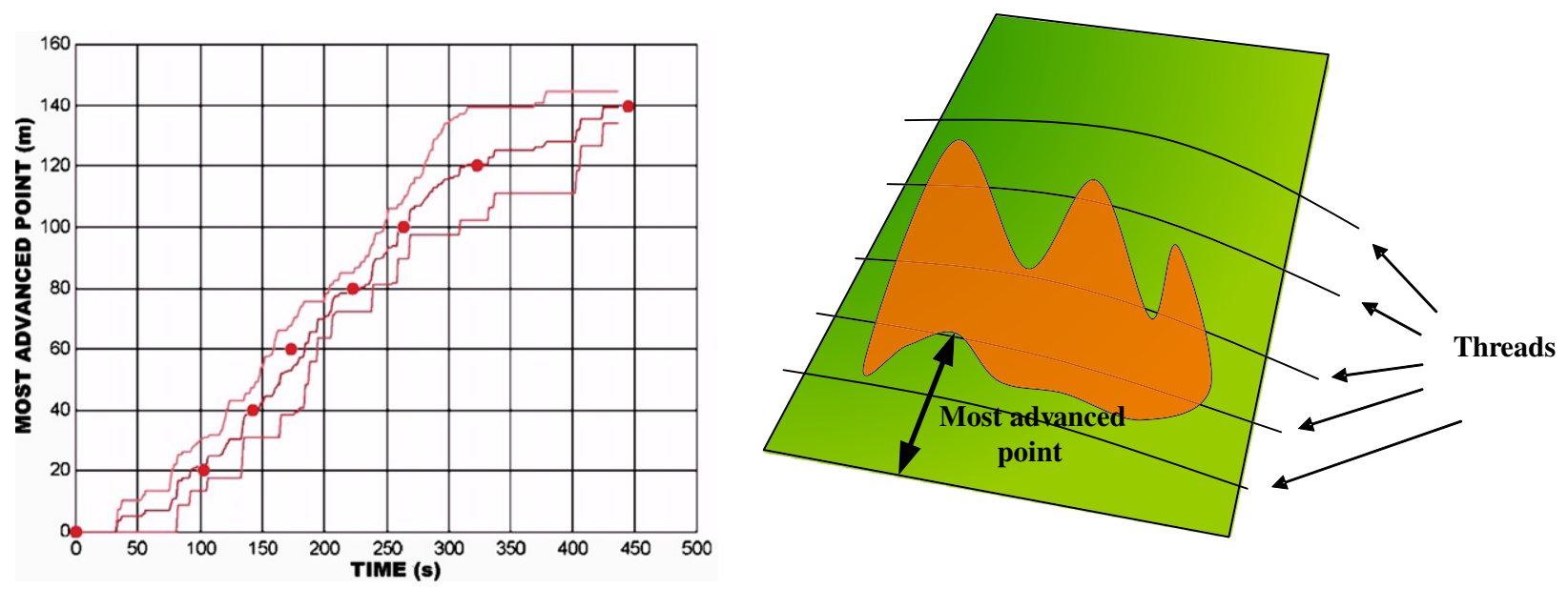

Fig. 19. Left: advance of a fire monitored using an infrared and a visual camera. Right: scheme of the threads used for manual computation of fire advance. 

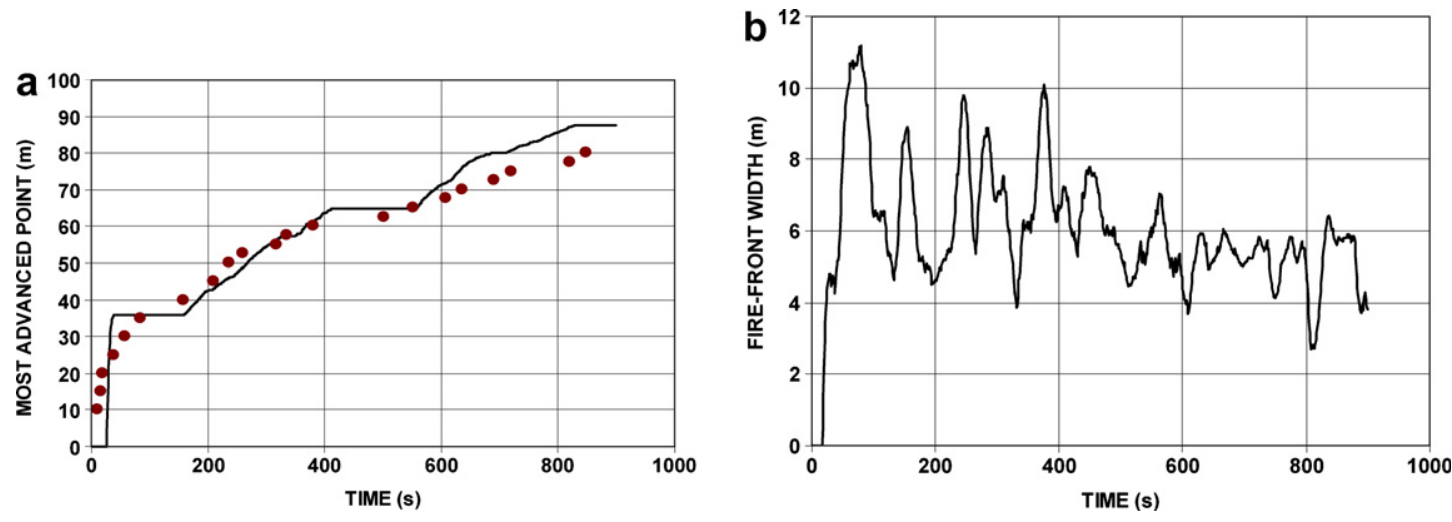

Fig. 21. (a) Advance of the fire front, (b) fire front width.

computes a 3D perception model of the fire, which can be transmitted via TCP/IP for its visualization in remote computer systems.

The paper includes some results of fire experiments carried out in Gestosa (near Coimbra, Portugal) since 1998. These experiments, close to operational conditions, have demonstrated the interest of the presented system, which is currently being used by research groups on fire behaviour. One of the main conclusions of these tests is that the robustness of the system against the potential sources of errors can be accomplished by using complementary information, provided by cameras of different types (visual and infrared) at different locations.

The experiments described in the paper have been carried out using mainly fixed cameras. The extraction of fire measures using cameras on vehicles and particularly on helicopters is object of current research $[16,13]$. On the other hand, new research and development activities have been planned for the operational use of the system in the INFOCA forest fire fighting plan of the Regional Government of Andalucía (Spain).

\section{Acknowledgements}

The work described in this paper was initially developed in the European projects SPREAD "Forest Fire Spread Prevention and Mitigation" (European Commission, 5th Framework Programme, EVG1-CT-2001-00043) and COMETS (5th Framework Programme, IST-2001-34304). The recent funding of the projects AEROSENS (Spanish Dirección General de Investigación DPI2005-02293), AWARE (6th Framework Programme, IST-2006-33579) and SADCON (Excellence Project of the Andalucía Regional Government) also contributed in the last part of this work. The authors thank the help of Fernando Prado, Carmen Rosa, Juan José Giraldo, Francisco Ortiz and Antonio Luis Flores in the development of the work. The Gestosa Experiments have been performed with the help and assistance of Prof. Viegas and his ADAI team at the University of Coimbra (Portugal). The authors also acknowledge the motivation and advice of Francisco Salas, head of the Forest Fire Prevention and Restoration Service of the Andalucía Regional Government, and currently at the company EGMASA in charge of the Environment Services in Andalucía.

\section{References}

[1] H. Amano, K. Osuka, T. Tzyh-Jong, Development of vertically moving robot with gripping handrails for fire fighting, Proceedings of the IEEE/RSJ International Conference on Intelligent Robots and Systems 2 (2001) 661-667.

[2] B.C. Arrue, A. Ollero, J.R. Martinez-de Dios, An intelligent system for false alarm reduction in infrared forest-fire detection, IEEE Intelligent Systems 15 (3) (2000) 64-73.

[3] A. Bardshaw, The UK Security and Fire Fighting Advanced Robot project, IEE Colloquium on Advanced Robotic Initiatives in the UK, London, UK, 1991.

[4] T.H. Coleman, Y. Li, On the convergence of reflective Newton methods for large-scale nonlinear minimization subject to bounds, Mathematical Programming 67 (N²) (1994) 189-224.

[5] T.H. Coleman, Y. Li, An interior, trust region approach for nonlinear minimization subject to bounds, SIAMM Journal on Optimization 6 (1996) 418-445.

[6] E. Den Breejen, M. Roos, K. Schutte, J.S. De Vries, H. Winkel, Infrared measures of energy release and flame temperatures of forest fires, Proceedings of third International Conference on Forest Fire Research, Luso (Portugal), 1998, pp. 517-532.

[7] D. Dierre, H. Hoff, M. Bouchet, RAPSODI, rapid smoke detection and forest fire control, Research Special Session 2000 (2000) 89-96.

[8] O. Faugeras, Q.-T. Luong, The Geometry of Multiple Images, The MIT Press, Cambridge, MA, 2001.

[9] A.H. Gunatilaka, B.A. Baertlein, Feature-level and decision-level fusion of non-coincidently sampled sensors for land mine detection, IEEE Transactions on Pattern Analysis and Machine Intelligence 23 (6) (2001) 577-589.

[10] J.R. Martinez-de Dios, A. Ollero, A multiresolution threshold selection method based on training, LNCS 3211 (2004) 90-97.

[11] J.R. Martinez-de Dios, A. Ollero, A multiresolution-Fuzzy method for robust threshold selection in image segmentation, Intelligent Automation and Soft Computing 12 (4) (2006) 419-430.

[12] L. Merino, F. Caballero, J.R. Martínez-de Dios, J. Ferruz, A. Ollero, A cooperative perception system for multiple UAVs: application to automatic detection of forest fires, Journal of Field Robotics 23 (2006) 165-184.

[13] L. Merino, A. Ollero, J. Ferruz, J.R. Martinez-de Dios, B. Arrue, . Motion analysis and geolocation for aerial monitoring in the COMETS multi-UAV system, Proceeding of the ICAR, Coimbra, (Portugal), 2003, pp. 351-356. 
[14] A. Mohamad-Djafari, Probabilistic methods for data fusion, Proceedings of the 17th International Workshop on Maximum Entropy and Bayesian Methods for Statistical Analysis, 1997, Boise, Idaho (USA).

[15] A. Ollero, B.C. Arrúe, J.R. Martínez, J.J. Murillo, Techniques for reducing false alarms in infrared forest-fire automatic detection systems, Control Engineering Practice 7 (1999) 123-131.

[16] A. Ollero et al., Control of multiple heterogeneous unmanned aerial vehicles: architecture and perception issues in the COMETS project, IEEE Robotics and Automation Magazine 12 (2005) 46-57.

[17] W. Philips, M. Shah, N. da Vitoria-Lobo, Flame recognition in video, Pattern Recognition Letters 23 (1-3) (2002) 319-327.

[18] T.W. Ridler, S. Calvard, Picture thresholding using an iterative selection method, IEEE Transactions on Systems, Man, and Cybernetics SMC-8 (1978) 630-632.
[19] J. San-Miguel-Ayanz, N. Ravail, V. Kelha, A. Ollero, Active fire detection for fire emergency management: potential and limitations for the operational use of remote sensing, Natural Hazards $n^{\circ} 35$ (3) (2005) 361-376.

[20] D.X. Viegas, M.G. Cruz, L.M. Ribeiro, A.J. Silva, A. Ollero, B. Arrue, R. Martínez-de-Dios, F. Gómez Rodríguez, L.Merino, A.I. Miranda, P. Santos, Gestosa fire spread experiments, Proceeding of the fourth International Congress on Forest Fire Research, Luso, (Portugal), 2002

[21] J.L. Wybo, G. Eftichidis, D. Koutsouris, T. Manganas, D.X. Viegas, T. Apostolopoulos, E. Pelosio, G. Bovio, A. Ollero, D. Schmidt, A. Criado, DEDICS: a general supporting management of forest fire, Proceeding of the third International Conference on Forest Fire Research, Luso (Portugal), 1998, pp. 2003-2012. 